\title{
Indirect effects among biodiversity loss of mutualistic ecosystems
}

\author{
Guangwei Wang ${ }^{1,2}$, Xueming Liu ${ }^{1}$, Guanrong Chen $^{3}$ \& Hai-Tao Zhang ${ }^{1,2, *}$ \\ ${ }^{1}$ School of Artificial Intelligence and Automation, Key Laboratory of Image Processing and Intelligent Control, Huazhong University of \\ Science and Technology, Wuhan 430074, China; \\ ${ }^{2}$ State Key Lab of Digital Manufacturing Equipment and Technology, Huazhong University of Science and Technology, Wuhan 430074, \\ China; \\ ${ }^{3}$ Department of Electrical Engineering, City University of Hong Kong, Hong Kong, China \\ *Corresponding author (email: zht@mail.hust.edu.cn)
}

Received: 7 December 2021; Revised: 21 January 2022; Accepted: 16 February 2022; Published online: 20 May 2022

\begin{abstract}
Drastic reduction in biodiversity has been a severe threat to ecosystems, which is exacerbated when losing few species leads to disastrous and even irreparable consequences. Therefore, revealing the mechanism underlying biodiversity loss is of uttermost importance. In this study, we show that abundant indirect interactions among mutualistic ecosystems are critical in determining species' status. Combining topological and ecological characteristics, we propose an indicator derived from a dynamic model to identify keystone species and quantify their influence, which outperforms widely-used indicators like degree in realistic and simulated networks. Furthermore, we demonstrate that networks with high modularity, heterogeneity, biodiversity, and less intimate interactions tend to have larger indirect effects, which are more amenable in predicting decline of biodiversity with the proposed indicator. These findings shed some light onto the influence of apposite biodiversities, paving the way from complex network theory to ecosystem protection and restoration.
\end{abstract}

Keywords: biodiversity, indirect effect, complex network, mutualism

\section{INTRODUCTION}

The sixth mass extinction episode [1] has been characterized by rapid rate of biodiversity loss [2] and extensive endangered species (the IUCN Red List of Threatened Species. https://www.iucnredlist.org/en) facing imminent crisis of annihilation compared with historical data [3]. Both marine and terrestrial ecosystems are confronting biodiversity and population decline, while minor biodiversity decline may have more devastating consequences such as massive secondary extinction [4]. Especially in ubiquitous mutualistic ecosystems, like those composed of plants and pollinators or seeds and dispersers, annihilation of a single species could trigger long-term shifts in systems' states due to the complex dynamic interactions. More seriously, such biodiversity decline could eventually drive a system to a degraded state and even disastrous disintegrate. The striking prospect of potential ecosystem decline [5] and extirpation [6] has motivated research on prediction of biodiversity loss as well as keystone species identification [7].

To investigate the importance of different species and forecast secondary extinctions, a topological coextinction model (TCM) [8-10] has been established according to static characteristics of ecosystems, where

(c) The Author(s) 2022.Published by China Science Publishing \& Media Ltd. and EDP Sciences. This is an Open Access article distributed under the terms of the Creative Commons Attribution License (https://creativecommons.org/licenses/by/4.0), which permits unrestricted use, distribution, and reproduction in any medium, provided the original work is properly cited. 
peripheral species with few direct interactions could surprisingly have significant influence. As for the criterion of species' annihilation, in TCM, a species dies out once losing all direct interactions, with advantages in both computational complexity and parameterize amenability. However, such prerequisite deprives inherent features of ecosystems, such as species' growth and mortality rate [11], diverse interaction strength [12], and ecologically effective population size (EEP) [13].

Incorporating species' various dependence on mutualistic interactions, stochastic coextinction model (SCM) $[7,12]$ introduces indirect additional losses. Hence, SCMs allow for more realistic and general eradication phenomena, where topologically irrelevant species associated by long paths are involved. Essentially, in SCMs, species' probabilities of coextinct are assigned instead of being developed according to topological or dynamical properties. In [7], the investigation is limited to species with high influence, postulating that less influential species seldom trigger secondary extinction. Due to sophisticated dynamic interactions such as mutualism and competition [14], the estimation accuracy is still restricted on system states, influential species, and biodiversity decline. Besides, in actual ecosystems, species are declared functionally extinct when their abundance falls below a certain EEP threshold and reduces biodiversity [15].

Previous experimental and theoretical studies have demonstrated that indirect effects are critical in maintaining persistence [16], determining composition [17], and guiding trait evolution [18] of ecosystems. To explore the determinant factors of individual species' influence mechanism on far reaching loss of biodiversity, both direct and underappreciated indirect effects should be investigated to elucidate how perturbation or influence of certain species propagate through complex ecosystems. Meanwhile species' abundance and systems' states should be tracked with dynamic models.

Nourished by more and more deep understanding of ecological interactions [19] and complex network theory [7], both direct and indirect effects in biodiversity and trait coevolution [18], species invasion [20], and secondary extinction [7] have recently drawn considerable attention. Enabled by network analyzation, the impact of ecosystems' structure and multiple interactions has been investigated on species' importance and information propagation rather than focusing on apparent direct interactions [7].

In this paper, we propose an indicator $T^{\text {out }}$ based on dynamic coextinction model (DCM) integrating topological, dynamical, and ecological properties to explore the influence of different species in mutualistic ecosystems, which greatly facilitates the keystone species identification and prediction of biodiversity decline. Applying the concept of information flow [18] and thresholds for functional extinction [19], we quantitatively evaluate both direct and indirect effects on the persistence of networks. Finally, each species' impact is explored through multiple interaction paths. Species' importance and biodiversity decline could thus be predicted.

\section{RESULTS}

\section{Model and total effects of mutualistic systems}

We analyze species' indirect effect and important indicator of ecosystems with 29 real networks covering four different kinds of mutualistic interactions, including plant-pollinator, seed-dispersal, plant-ant, and anemonefish (Supplementary Table S1). We also constructed simulated weighted networks to validate the indicator (Supplementary Figure S2). We illustrate the observations by a medium-sized network $M_{-} S D_{-} \_03$, which 
includes 25 plants, 16 dispersals, and 68 interactions obtained from Caguana, Puerto Rico.

We use a dynamic model of bipartite mutualistic networks [14] to track the evolution of species abundance. $P_{i} / A_{i}$ is the abundance of the $i$ th species of different groups, such as plant/pollinator, and each group is composed of $N_{P} / N_{A}$ species:

$$
\left\{\begin{array}{l}
\frac{\mathrm{d} P_{i}}{\mathrm{~d} t}=P_{i}\left(\alpha_{i}^{(P)}-\sum_{j}^{N_{P}} \beta_{i j}^{(P)} P_{j}+\frac{\sum_{j}^{N_{A}} \gamma_{i j}^{(P)} A_{j}}{1+h \sum_{j}^{N_{A}} \gamma_{i j}^{(P)} A_{j}}\right), \\
\frac{\mathrm{d} A_{i}}{\mathrm{~d} t}=A_{i}\left(\alpha_{i}^{(A)}-\sum_{j}^{N_{A}} \beta_{i j}^{(A)} A_{j}+\frac{\sum_{j}^{N_{P}} \gamma_{i j}^{(A)} P_{j}}{1+h \sum_{j}^{N_{P}} \gamma_{i j}^{(A)} P_{j}}\right),
\end{array}\right.
$$

$\alpha$ is the intrinsic growth rate in the absence of interaction or perturbation. Intraspecific $\left(\beta_{i i}\right)$ and interspecific $\left(\beta_{i j}\right)$ competition reflect environmental conditions, generally $\beta_{i i} \gg \beta_{i j}(i \neq j)$. The profit that a species could obtain from between-group mutualistic interactions is restricted by the half-salutation constant $h$, corresponding to ecological interaction constraints. Parameter $\gamma$ is the specific interaction strength with

$$
\gamma_{i j}=\varepsilon_{i j} \frac{\gamma_{0}}{\left(K_{i}\right)^{t}}
$$

in the binary matrix $\varepsilon$, if node $i$ directly interacts with node $j$ then $\varepsilon_{i j}=1, \gamma_{i j}$ quantifies the mutualistic strength; otherwise $\varepsilon_{i j}=0, \gamma_{i j}=0$. Specifically, $\gamma_{0}$ is taken from a uniform distribution [21]: $\gamma_{0} \sim U(0,1)$. $K_{i}$ is the amount of mutually beneficial interaction of species $i$, and $t$ measures the tradeoff between topological and ecological properties, representing how the amount of interaction mediates the overall mutualistic strength. Notably, species with abundance below a certain EEP threshold or getting disconnected from the largest connected component are considered to be extinct [22-24], standing for functional extinction phenomena, and to exclude discrete nodes and minor sub-clusters to ensure statistical significance [19,25].

We first derive a $\boldsymbol{\Gamma}$-matrix to describe the direct profitable mutualistic effect (Figure 1A):

$$
\boldsymbol{\Gamma}=\left[\begin{array}{cc}
0 & \gamma^{(P)} \\
\gamma^{(A)} & 0
\end{array}\right],
$$

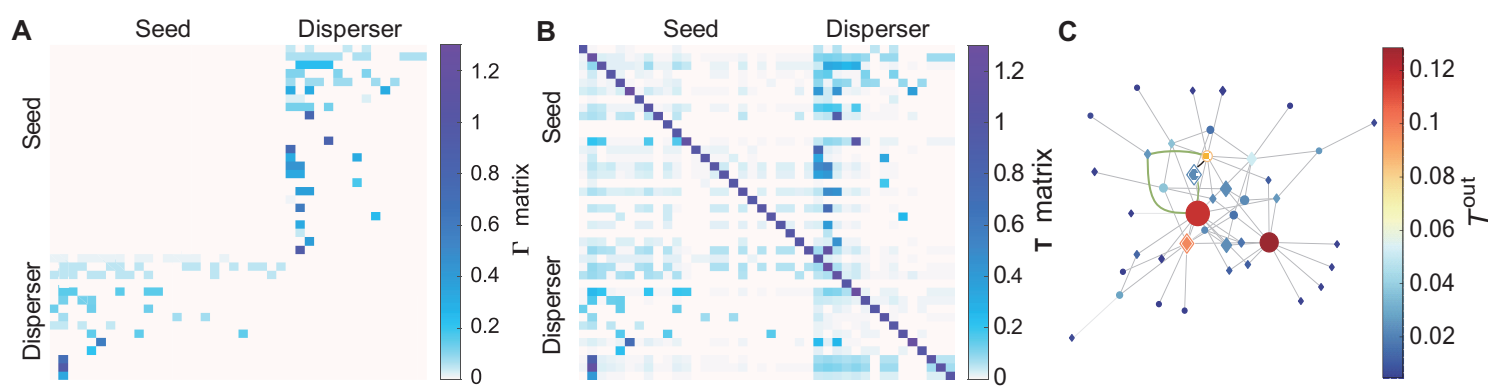

Figure 1 Both direct and indirect mutualistic effects and each species' total impact on a seed-dispersal mutualistic network $M_{-} S D_{-} 003$. (A) and (B) Quantitative direct benign mutualistic effects ( $\boldsymbol{\Gamma}$-matrix) and overall influence of different species on others through both direct and indirect interactions (T-matrix). Purple dots in the matrix indicate the species in the column have a larger influence on species in the row, whereas white dots suggest weaker influence. (C) The structure of the bipartite network, where sizes, types, and colors of nodes indicate their degrees, classes, and $T^{\text {out }}$ s: larger nodes have more direct interactions. Diamonds denote plants while circles are dispersers; nodes with warmer color exert greater impact on the network through multiple paths. Among the nodes, we highlight two white-edged nodes that have identical degrees but have varying $T^{\text {out }}$ values, i.e., total influence including direct and indirect effects. For two patterned nodes, we indicate their direct interaction in black, and one of the indirect interactions in green. 
where $\gamma^{(P)}$ and $\gamma^{(A)}$ are composed of $\gamma_{i j}^{(P)}$ and $\gamma_{i j}^{(A)}$ in eq. (1), indicating how species benefit from direct interactions through paths with length $l=1$ (highlighted as black path in Figure 1C). As for topologically non-interacting species, nonintuitive indirect effects spreading through paths with $l>1$ serve momentous roles in the system thriving ( $l=3$ for the highlighted green indirect path in Figure 1C). To quantitatively estimate the total influence, we combine the numerous multi-paths interactions and consider all indirect paths' lengths as $l=2,3, \ldots, n$. If species $i$ and $j$ interact via paths of length $l$ then $\Gamma_{i j}^{l}>0$, which form the $\Gamma^{l}$ matrix representing $l$-order indirect mutualistic effects [7]. By setting $t=1$ in eq. (2), that is network topology affects mutualistic effects through both direct and indirect interactions, for each row of $\boldsymbol{\Gamma}$, we have

$$
\sum_{j}^{N} \Gamma_{i j}<1,
$$

where the network size $N=N_{P}+N_{A}$, and the real part of the leading eigenvalue satisfies $0<\lambda_{\Gamma}<1$. Thus, all the direct and indirect effects can be described by a matrix $\mathbf{T}$ (Figure 1B) as follows:

$$
\mathbf{T}=\boldsymbol{\Gamma}^{0}+\boldsymbol{\Gamma}^{1}+\boldsymbol{\Gamma}^{2}+\cdots+\boldsymbol{\Gamma}^{\infty}=\sum_{l=0}^{\infty} \boldsymbol{\Gamma}^{l}=(\mathbf{I}-\boldsymbol{\Gamma})^{-1},
$$

where $\mathbf{I}$ is the identity matrix. $T_{i j}$ describes species $i$ 's impact on species $j$ through direct and indirect paths, and the indicator of total influence of species $i$ in the system (Figure 1C) is

$$
T_{i}^{\text {out }}=\frac{1}{N} \sum_{j}^{N} T_{i j} .
$$

The indirect effect $\psi_{i}$ of each species $i$ can be evaluated by

$$
\psi_{i}=\frac{\sum_{j}^{N} T_{i j} \times\left(1-\varepsilon_{i j}\right)}{\sum_{j}^{N} T_{i j}} .
$$

We finally obtain the system's overall indirect effects as

$$
\Psi=\frac{\sum_{i}^{N} \sum_{j}^{N} T_{i j} \times\left(1-\varepsilon_{i j}\right)}{\sum_{i}^{N} \sum_{j}^{N} T_{i j}} .
$$

\section{Quantifying species' indirect effect}

Although lacking accurate estimation, indirect effects play an indispensable role in spreading benefits of interactions and diffusing local perturbations. In Figure 2A, we show species' indirect effect on different mutualistic ecosystems. Counterintuitively, species with few apparent direct beneficial interactions may have profound indirect effects on other species [26], whose effects are not necessarily aligned with direct topological features like degree as shown in Figure 2B. Generalists with abundant interactions may not be as critical as they appear to be, and hub nodes may not be the most influential species either. Species with the same amount of direct interactions are of various importance due to those impacts passed through complicated indirect paths, as shown in Figure 1C. Hence, considering only direct interactions of species is not sufficient to accurately predict their importance or profound influence once obliterated. A thorough niche indicator as $T^{\text {out }}$ derived from the DCM in eq. (6) becomes essential. 

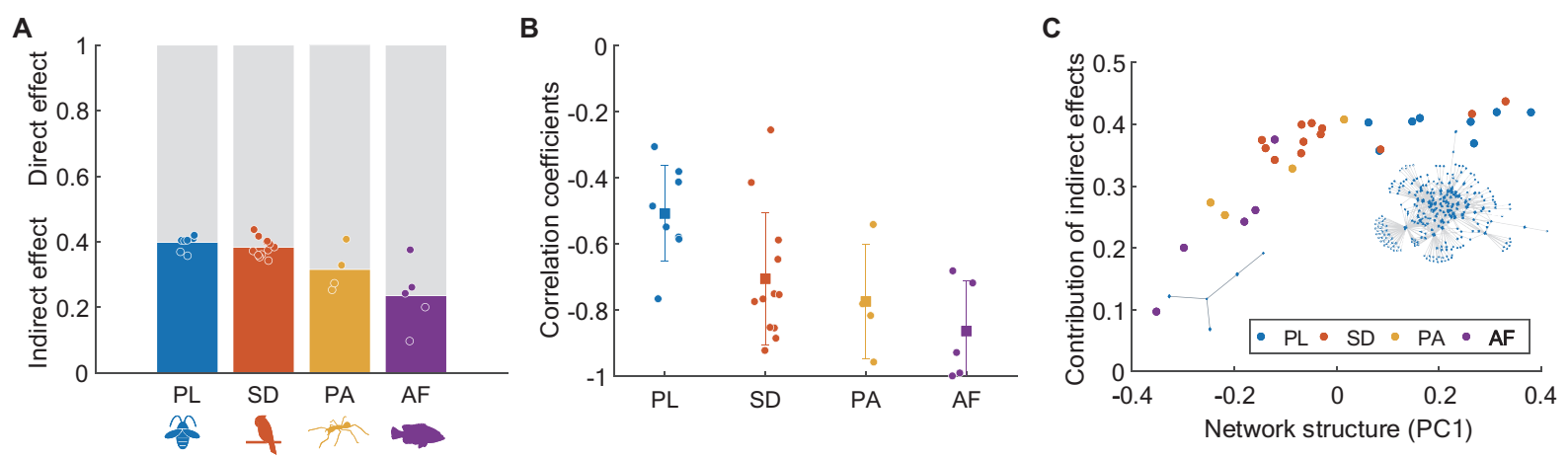

Figure 2 Determinants of indirect effects. (A) Scatters are indirect effects of different types of mutualistic ecosystems; grey bars and colored bars show average direct and indirect effects, respectively. (B) For the majority of all four types of networks, species' indirect effects have no significant relation to their degrees (especially PL and SD, $p<0.001$ ), while in some networks (PA and AF) species' indirect effects are negatively correlated with their degrees. (C) Indirect effects of multi-types of mutualistic networks increase along the gradient based on PC1 of principal component analysis, where well connected and nested networks (negative PC1 values) have small indirect effects, and modular, heterogeneous, large networks (positive PC1 values) have large indirect effects. The two networks in (C) show different structures (PL/AF) with large/small PC1 values and indirect effects, respectively. In (A)-(C), different colors represent different mutualistic networks: PL: plant-pollinator, SD: seed-dispersal, PA: plant-ant, AF: anemone-fish. Parameters: $\alpha_{i}=0.5, \beta_{i i}=1, \beta_{i j}=0, h=0.2$, threshold $\theta=0.001$.

Corresponding to topological characteristics, we perform a principal component analysis (PCA) [18, 27] with five structural descriptors (Figure 2C, Methods), which together leads to the conclusion that indirect effect has greater influence on networks with lower connectivity and nestedness, and higher modularity, heterogeneity and biodiversity.

From the ecological points of view, more intimate mutualistic interactions create smaller indirect effect, such as anemone-fish ecosystems containing mostly specialized and obligate mutualistic interactions [28,29], while plant-pollinator ecosystems have more generalists and thence more redundancy [8].

\section{Identifying keystone species and predicting biodiversity loss}

Accordingly, we assess species' importance by the virtue of indicator $T^{\text {out }}$. In this way, we could identify keystone species and then predict biodiversity decline after losing certain species, which may be triggered by overexploitation, habitat fragmentation, and environmental degradation.

With poverty of data and limited knowledge of ecosystems, topological keystone species indicators [7,10] may be used to provide some rough estimations. However, ecosystems' decisive nature such as mutualism, competition, and antagonism that distinguishes networks of similar structures is usually overlooked, not to mention the species topologically analogous to each other that behave discrepantly. Hence, it is essential to bring dynamics to the estimation of species' abundance and systems' states, while considering the dominant interactions and the mutualistic effects on preserving biodiversity. Thus, we adopt the bipartite high-dimensional coupled model (eq. (1)) to track the variation of species' abundance when randomly removing species, and apply the proposed indicator $T^{\text {out }}$ in eq. (6) to take into account both self-dynamics and benefits of mutualistic interactions.

We first verify whether $T^{\text {out }}$ and species-level topological descriptors can assess species' importance and predict secondary extinction (Figures 3A and 3B, Supplementary Table S2) when eliminating single species. While fitting biodiversity loss and different indicators with a linear model (Figure 3C), $T^{\text {out }}$ shows better 
A

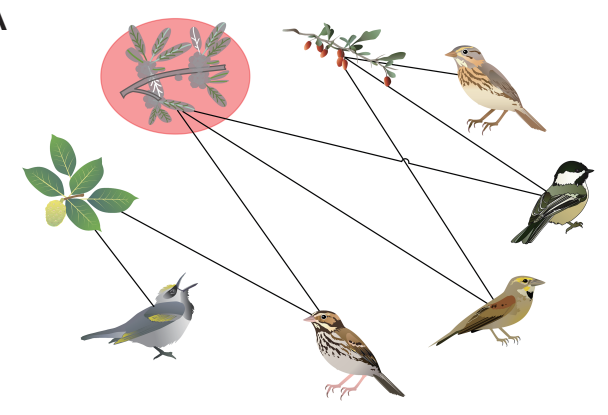

C

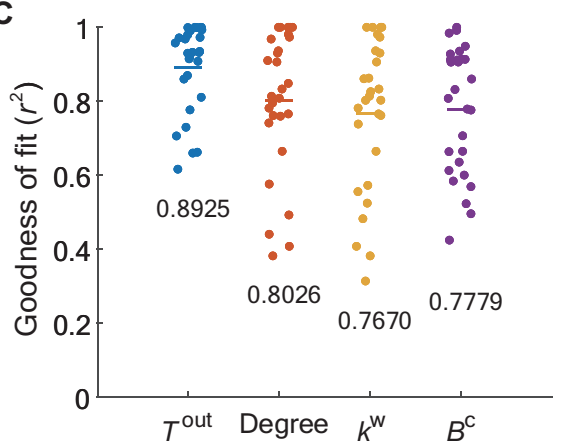

B

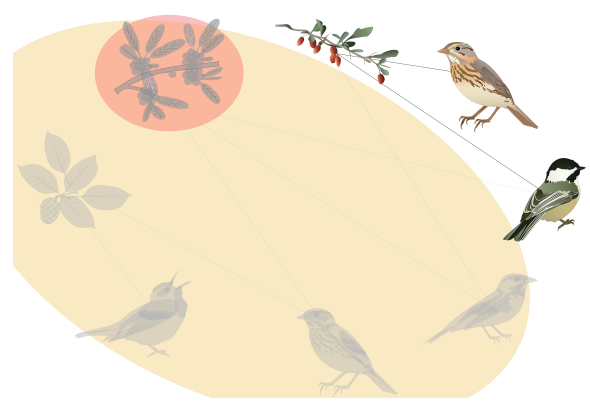

D

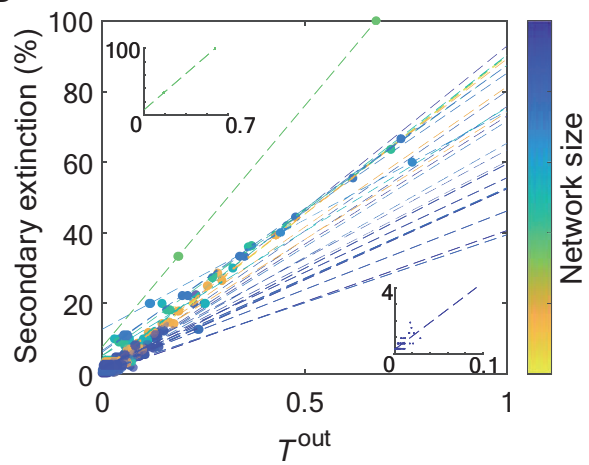

Figure 3 Relationship between the biodiversity loss and different indicators. (200 independent simulation runs) (A) and (B) The secondary extinction in an 8-component seed-disperser ecosystem. Removing species leads to massive biodiversity and interaction loss. Here, the local ecosystem may suffer remarkable degradation as shown in (B) compared with the original state. (C) Fitting secondary extinction size and $T^{\text {out }}$, degree, $k^{\mathrm{w}}$ (weighted degree), $B^{\mathrm{c}}$ (betweenness centrality) with a linear model after removing a single species. (D) Species with higher impact on the network (larger $T^{\text {out}}$ ) have larger disposition to induce massive secondary extinction. Dashed lines in different colors show the fitted linear model of different networks, which show the size evolution of secondary extinction along increasing $T^{\text {out }}$. Here colder color suggests larger networks while warmer color represents the smaller ones. The smaller pictures show two fitting curves with small and large slopes, which indicate small and large secondary extinction trends, respectively.

fitness compared with straight forward topological indicators according to adjusted coefficient of determination (adjusted- $r^{2}$ ). Akaike information criterion (AIC) is also used to compare their performances (Supplementary Table S3). Here, degree [30] describes biotic interspecific interactions and potential of spreading perturbations to directly interacting species. Weighted degree $k^{\mathrm{w}}$ represents both nodes' interactions and strengths [31]. Degrees also reflect species' importance in TCMs. Betweenness centrality [32] is the amount of shortest paths between any two nodes that pass through a certain node, illustrating how removing certain species may alter the path lengths.

In Figure 3D, removing species with lower $T^{\text {out }}$ results in fewer and minor secondary extinctions, thus $T^{\text {out }}$ serves as a niche indicator of keystone species. Species with large $T^{\text {out }}$ values are more likely to trigger massive secondary extinction, which makes them crucial in the maintenance and conservation of ecosystems.

\section{Biodiversity loss in more general scenarios}

In real scenarios, multiple species may go extinct simultaneously due to environmental deterioration [1]. We mimic such more general situations by removing a fraction $f_{n}$ of species from the ecosystem.

While simultaneously removing multiple species (Figure 4), we estimate the total influence of the removed 
A

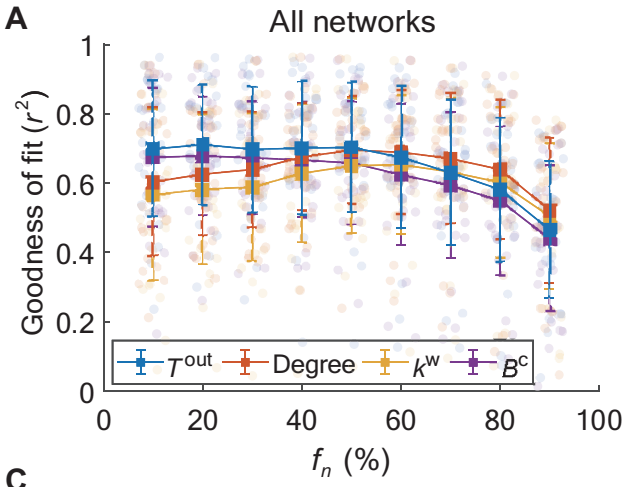

C

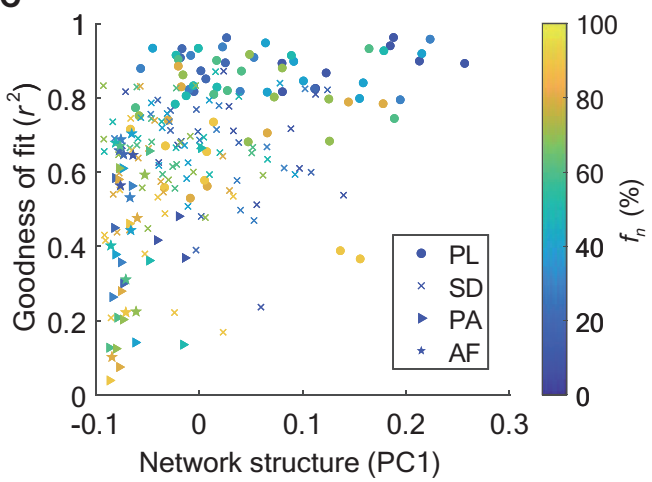

B

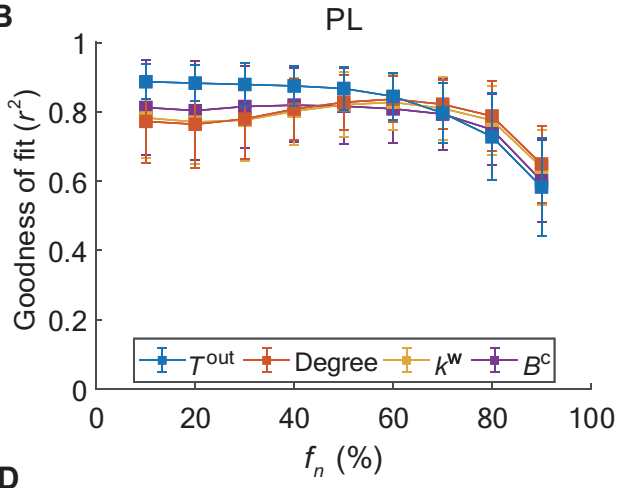

D

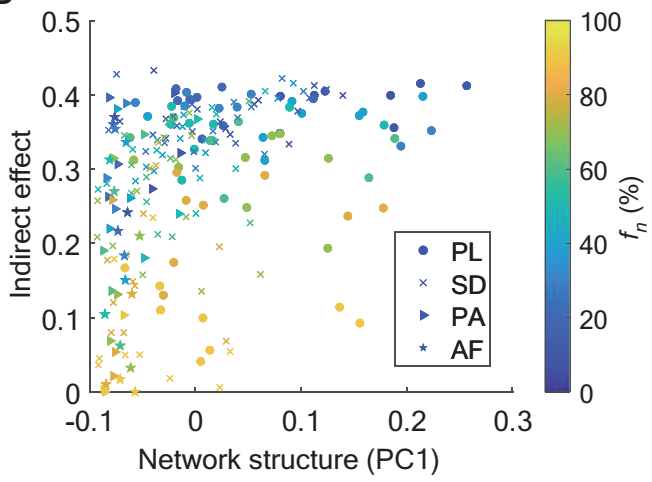

Figure 4 Biodiversity loss and different indicators in more general scenarios. (200 independent simulation runs) (A) Fitness of the linear model relating the secondary extinction size and different indicators $T^{\text {out }}$, degree, $k^{\mathrm{w}}$ (weighted degree), $B^{\mathrm{c}}$ (betweenness centrality) with increasing removal range of species of all the 29 networks. As shown in (B), the result of PL (plant-pollinator) networks has high indirect effect and increasing removal range. (C) and (D) are the relationship between secondary extinction sizes and $T^{\text {out }}$. (C) Capacity of indicating

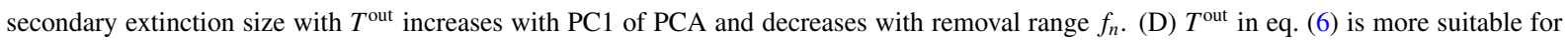
networks with higher indirect effect (and small removed range). Different markers correspond to four different types of ecosystems, where colorbars show the percentage of removed species.

species by summing their individual impacts. Indicators can be applied to predict secondary extinction in networks such as those composed of plants and pollinators. However, in certain scenarios all the indicators fail to predict the exact consequence with a fitted linear model.

We apply PCA to figure out under what circumstances the proposed indicator $T^{\text {out }}$ can properly indicate the biodiversity decline. As shown in Figures $4 \mathrm{C}$ and $4 \mathrm{D}, T^{\text {out }}$ is available to networks with low connectivity and nestedness, and high modularity, heterogeneity and biodiversity. That is, loss of biodiversity in networks with higher indirect effects (Figure 4D) can be better predicted, for example in plant-pollinator ecosystems (Figure 4B and Supplementary Figure S1). By contrast, networks with high connectivity and nestedness do not incline to be dominated by indirect effects. A typical example is the anemone-fish networks where more species complete life stages with few interactions [33]. Along with the decrease of indirect effects, the direct effects claim the primary responsibility and hence indicators reflecting such impact are more effective in predicting biodiversity decline, such as the degree after removing $95 \%$ of species.

Significantly, as removal range expands, there exists a critical removal fraction beyond which pruning species leads to system disintegration. For all four types of networks, such a debacle does not happen until the majority of species are stochastically removed (Figure 5). In spite of their robustness to random extermination [34], systems' critical removal fraction of disintegrating becomes much smaller once the species 


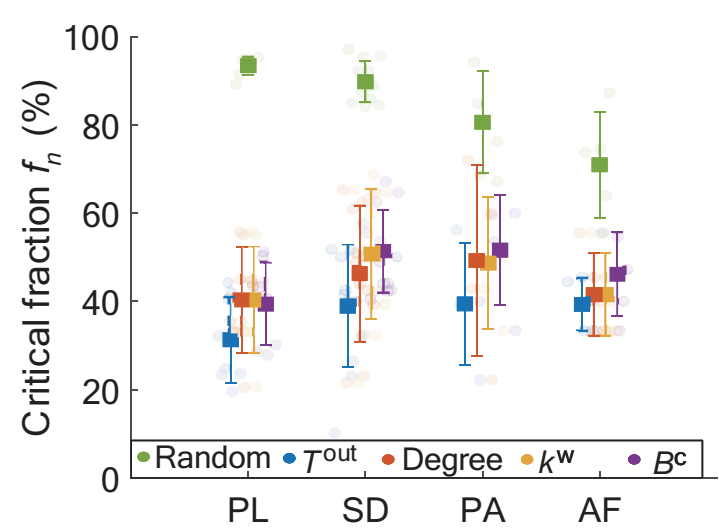

Figure 5 Critical removal fraction of networks under random and intentional removal. In different kinds of network, the critical removal fraction is much lower when targeting keystone species identified by different indicators compared with random removal, especially $T^{\text {out }}$. Different colors suggest different indicators.

are intentionally removed according to $T^{\text {out }}$ or other aforementioned topological indicators, among which removing keystone species identified by $T^{\text {out }}$ is prone to cause much rapid declination. Such targeted removals disintegrate networks much more efficaciously than random removal, demonstrating the reliability of the indicators derived from DCM. Moreover, the proposed indicator $T^{\text {out }}$ shows a high precision for predicting keystone species, which could help optimize reservation and restoration strategies $[35,36]$ with limited resources in urgent situations of ecosystems.

\section{DISCUSSION}

Incorporating network dynamics and ecological characteristics, we identify keystone species and explore biodiversity decline of mutualistic networks with DCM. The TCMs [8-10] provide an insight of complex systems' secondary extinction, while reducing divergence of different interactions and species. As a step towards systems' dynamic evolution, SCMs cooperate with variation of mutualistic interactions and species' dependence [12]. Nevertheless, restricted by scarce empirical observation and lack of adequate understanding of ecosystems [7], SCMs randomly assign coextinction probabilities. Here, DCM provides a more comprehensive description of dynamic and biodiversity evolution which is preferable for coupled high-dimensional ecosystems analysis.

The proposed indicator $T^{\text {out }}$ captures both topological and ecological features of mutualistic ecosystems. Combining indirect effects of ecosystems completes the propagation of species influence and perturbations through all possible paths in a network, which have been reckoned necessary in previous research and affirmed in our study. We found that indirect effects have profound influences on the prosperity or degradation, even disintegration of ecosystems, especially modular, heterogeneous, and large networks, typically plantpollinator ecosystems. In networks that possess more generalists and tend to share multiple interactions, indirect effects are more pervasive through extensive paths for which indicator $T^{\text {out }}$ becomes more applicable. We also compare the critical removal fraction of different types of ecosystems when removing several species in different scenarios, which substantiates the effectiveness of the index $T^{\text {out }}$ on quantification of species' importance and identifying keystone species. 
In conclusion, the present study sheds some light onto the connection between topological characteristics, dynamics of complex interactions, and biodiversity variation in mutualistic ecosystems. By integrating both direct and indirect effects of networks, we propose a methodology for distinguishing keystone species as well as predicting biodiversity decline in mutualistic networks. This methodology is more appropriate for less intimate mutualistic ecosystems with low connectivity and nestedness, and high indirect effects, modularity, heterogeneity and biodiversity. Our results identify invaluable species that may require extra protection or take precedence for ecosystems under threat, establishing potential guidelines in setting conservation priorities for protection and even restoration of endangered ecosystem.

\section{METHODS}

\section{Principal component analysis}

We conduct PCA to identify what kinds of network structures contain larger indirect effects and have better effects indicating secondary extinction size according to $T^{\text {out }}$. PCA has been a most common technique to reduce the pristine multivariate data to a small number of dimensions while preserving variability and increasing interpretability. Here, we investigate five structural descriptors of 29 mutualistic networks, i.e., connectivity, nestedness, modularity, heterogeneity, and size.

When analyzing the impact of indirect effect in an ecosystem, the first principal component PC1 contains $69.25 \%$ of the variation, and is negative with connectivity $(-0.4993)$ and nestedness $(-0.1696)$, but positive with modularity (0.1315), heterogeneity (0.5629), and size (0.6227). As shown in Figure 2C, indirect effect has greater influence on networks with low connectivity and nestedness, and high modularity, heterogeneity and biodiversity.

When removing multiple species from an ecosystem, we use PCA to find in what scenarios the proposed indicator $T^{\text {out }}$ can properly indicate decline of biodiversity. As shown in Figure 4C, the first principal component $\mathrm{PC} 1$ contains $71.27 \%$ of the variation, which is negative with connectivity $(-0.3777)$ and nestedness $(-0.0230)$, and positive with modularity $(0.2788)$, heterogeneity $(0.6009)$, and size $(0.6465)$. It implies that $T^{\text {out }}$ is suitable to indicate species contribution and coextinction size for networks with low connectivity and nestedness, and high modularity, heterogeneity and biodiversity.

\section{Information on mutualistic networks used in the study}

Here, the size of networks is the number of species they contain, for example, the size of a plant $(P)$ pollinator $(A)$ network $N$ is $N=N_{P}+N_{A}$. Network connectivity $C$ is $C=\sum_{i}^{N} \sum_{j}^{N} \varepsilon_{i j} /\left(N_{P} \times N_{A}\right)$, where $\varepsilon$ is the interaction matrix. Modularity indicates the presence of dense clusters of related nodes embedded in the network, which is calculated using the BiMat package (BiMat: http://bimat.github.io/). Nestedness describes the extent to which interactions form ordered subsets, and is calculated by the NODF measure. Network heterogeneity [19] $\mathcal{H}$ is $\mathcal{H}=\sigma_{\text {in }} \sigma_{\text {out }} /\langle s\rangle$, where $\langle s\rangle=\left\langle s^{\text {in }}\right\rangle=\left\langle s^{\text {out }}\right\rangle$ is the average incoming or outgoing weighted degree, $\sigma_{\text {in }}$ and $\sigma_{\text {out }}$ are variances of marginal probability density functions $P\left(s^{\text {in }}\right)$ and $P\left(s^{\text {out }}\right)$, respectively.

Topological indicators are derived as follows: species' degree is the number of direct interactors, degree $_{i}=$ $\sum_{j}^{N} \varepsilon_{i j}$; species' weighted degree [7] is $k_{i}^{w}=\operatorname{degree}_{i}^{1 / 2}\left(\sum_{j}^{N} A_{i j}\right)$, where $A$ is the weighted interaction matrix; 
betweenness centrality [32] is calculated as $B^{\mathrm{c}}(i)=\sum_{s \neq i, s \neq t, i \neq t} g_{s t}(i) / g_{s t}$, where $g_{s t}$ is the number of shortest paths between two nodes $s$ and $t$, and $g_{s t}(i)$ denotes the number of those shortest paths that pass through node $i$.

\section{Data availability}

Data on mutualistic networks are available from the Web of Life database (www.Web-of-Life.es). Other data and codes are available from the corresponding author upon reasonable request.

\section{Funding}

This work was supported in part by the National Natural Science Foundation of China (U2141235 and 61803166) and National Natural Science Foundation of Hubei Province (2019CFA005).

\section{Author contributions}

H.-T.Z. and G.R.C. conceived the project. X.M.L., H.-T.Z., and G.W.W. participated in the design of the study. G.W.W. and H.-T.Z. performed computations and data analysis, and drafted the manuscript. X.M.L., H.-T.Z., and G.R.C. revised the manuscript.

\section{Conflict of interest}

The authors declare that they have no conflict of interest.

\section{Supplementary information}

The supporting information is available online at https://doi.org/10.1360/nso/20220002. The supporting materials are published as submitted, without typesetting or editing. The responsibility for scientific accuracy and content remains entirely with the authors.

\section{References}

1 Ceballos G, Ehrlich PR, Dirzo R. Biological annihilation via the ongoing sixth mass extinction signaled by vertebrate population losses and declines. Proc Natl Acad Sci USA 2017; 114: E6089-E6096.

2 Gibb H, Silvey CJ, Robinson C, et al. Experimental evidence for ecological cascades following threatened mammal reintroduction. Ecology 2021; 102: e03191.

3 Barnosky AD, Matzke N, Tomiya S, et al. Has the Earth's sixth mass extinction already arrived? Nature 2011; 471: 51-57.

4 Ebenman B, Law R, Borrvall C. Community viability analysis: The response of ecological communities to species loss. Ecology 2004; 85: 2591-2600.

5 Jackson JBC, Kirby MX, Berger WH, et al. Historical overfishing and the recent collapse of coastal ecosystems. Science 2001; 293: 629-637.

6 Pimm SL, Raven P. Extinction by numbers. Nature 2000; 403: 843-845.

7 Pires MM, O'Donnell JL, Burkle LA, et al. The indirect paths to cascading effects of extinctions in mutualistic networks. Ecology 2020; 101: e03080.

8 Memmott J, Waser NM, Price MV. Tolerance of pollination networks to species extinctions. Proc R Soc Lond B 2004; 271: 2605-2611.

9 Rezende EL, Lavabre JE, Guimarães PR, et al. Non-random coextinctions in phylogenetically structured mutualistic networks. Nature 2007; 448: 925-928.

10 Pocock MJO, Evans DM, Memmott J. The robustness and restoration of a network of ecological networks. Science 2012; 335: 973-977. 
11 Rodríguez-Rodríguez MC, Jordano P, Valido A. Hotspots of damage by antagonists shape the spatial structure of plantpollinator interactions. Ecology 2015; 96: 2181-2191.

12 Vieira MC, Almeida-Neto M. A simple stochastic model for complex coextinctions in mutualistic networks: Robustness decreases with connectance. Ecol Lett 2015; 18: 144-152.

13 Säterberg T, Sellman S, Ebenman B. High frequency of functional extinctions in ecological networks. Nature 2013; 499: 468-470.

14 Rohr RP, Saavedra S, Bascompte J. On the structural stability of mutualistic systems. Science 2014; $345: 416$.

15 Estes JA, Terborgh J, Brashares JS, et al. Trophic downgrading of planet earth. Science 2011; 333: 301-306.

16 Rodriguez-Cabal MA, Noelia Barrios-Garcia M, Amico GC, et al. Node-by-node disassembly of a mutualistic interaction web driven by species introductions. Proc Natl Acad Sci USA 2013; 110: 16503-16507.

17 Novak M, Yeakel JD, Noble AE, et al. Characterizing species interactions to understand press perturbations: What is the community matrix? Annu Rev Ecol Evol Syst 2016; 47: 409-432.

18 Guimarães Jr PR, Pires MM, Jordano P, et al. Indirect effects drive coevolution in mutualistic networks. Nature 2017; 550: 511-514.

19 Gao J, Barzel B, Barabási AL. Universal resilience patterns in complex networks. Nature 2016; 530: $307-312$.

20 Holway DA, Lach L, Suarez AV, et al. The causes and consequences of ant invasions. Annu Rev Ecol Syst 2002; 33: 181-233.

21 Lever JJ, van Nes EH, Scheffer M, et al. The sudden collapse of pollinator communities. Ecol Lett 2014; 17: 350-359.

22 Watts DJ, Strogatz SH. Collective dynamics of 'small-world' networks. Nature 1998; 393: 440-442.

23 Fan C, Zeng L, Sun Y, et al. Finding key players in complex networks through deep reinforcement learning. Nat Mach Intell 2020; 2: 317-324.

24 Santolini M, Barabási AL. Predicting perturbation patterns from the topology of biological networks. Proc Natl Acad Sci USA 2018; 115: E6375-E6383.

25 Mello MAR, Felix GM, Pinheiro RBP, et al. Insights into the assembly rules of a continent-wide multilayer network. Nat Ecol Evol 2019; 3: 1525-1532.

26 Paine RT. A note on trophic complexity and community stability. Am Natist 1969; 103: 91-93.

27 Jackson JE. A User's Guide to Principal Components. Hoboken: John Wiley \& Sons. 1991

28 Frisch AJ, Rizzari JR, Munkres KP, et al. Anemonefish depletion reduces survival, growth, reproduction and fishery productivity of mutualistic anemone-anemonefish colonies. Coral Reefs 2016; 35: 375-386.

29 Figueiredo J, Baird AH, Connolly SR. Synthesizing larval competence dynamics and reef-scale retention reveals a high potential for self-recruitment in corals. Ecology 2013; 94: 650-659.

30 Lü L, Zhou T, Zhang QM, et al. The H-index of a network node and its relation to degree and coreness. Nat Commun 2016; 7: 10168.

31 Opsahl T, Agneessens F, Skvoretz J. Node centrality in weighted networks: Generalizing degree and shortest paths. Soc Networks 2010; 32: 245-251.

32 Newman MEJ. The structure and function of complex networks. SIAM Rev 2003; 45: 167-256.

33 Fontaine C, Guimarães Jr PR, Kéfi S, et al. The ecological and evolutionary implications of merging different types of networks. Ecol Lett 2011; 14: 1170-1181.

34 Srinivasan UT, Dunne JA, Harte J, et al. Response of complex food webs to realistic extinction sequences. Ecology 2007; 88: 671-682.

35 Sanhedrai H, Gao J, Schwartz M, et al. Reviving a failed network via microscopic interventions. 2020. ArXiv: 2011.14919

36 Ma C, Korniss G, Szymanski BK, et al. Universality of noise-induced resilience restoration in spatially-extended ecological systems. Commun Phys 2021; 4: 262. 